\title{
áridos
}

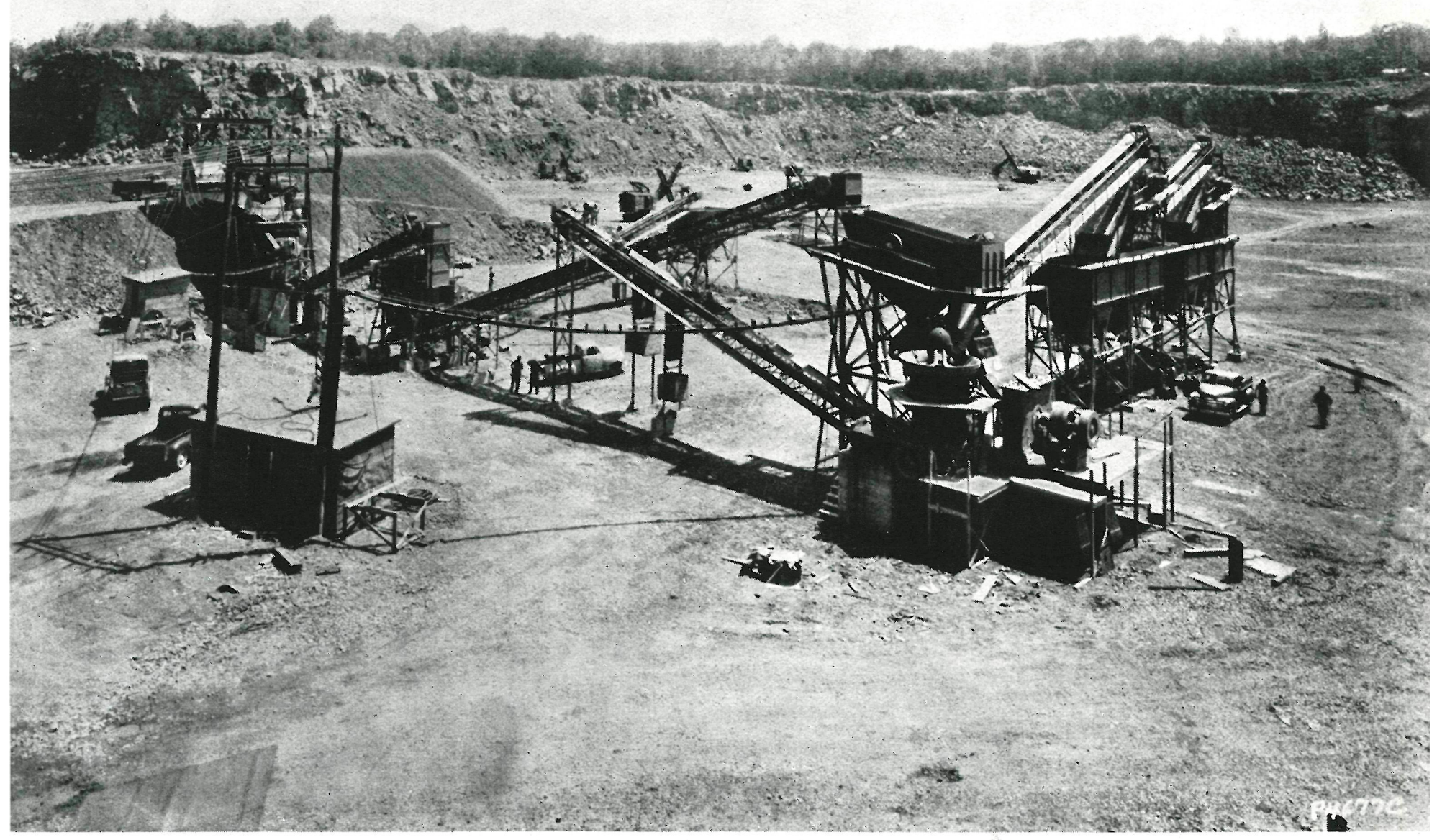

$866 \cdot 11$

\section{centrales de preparación y clasificación}

Información amablemente facilitada por la casa Pioneer Engineering Worlis, Inc. de Himneapolis (EL. IT.

ISTITUTO TECNICO DE LA JNSTRUCCION Y DEL CEMENTO
S I N O P S I S

En este trabajo se exponen una serie de ideas generales sobre la preparación y clasificación en centrales, así como la descripción de uno de los tipos normales de instalaciones de esta naturaleza, que ha sido proyectado por la casa Pioneer Engineering Works, especializada en el estudio y montaje de esta clase de instalaciones. 


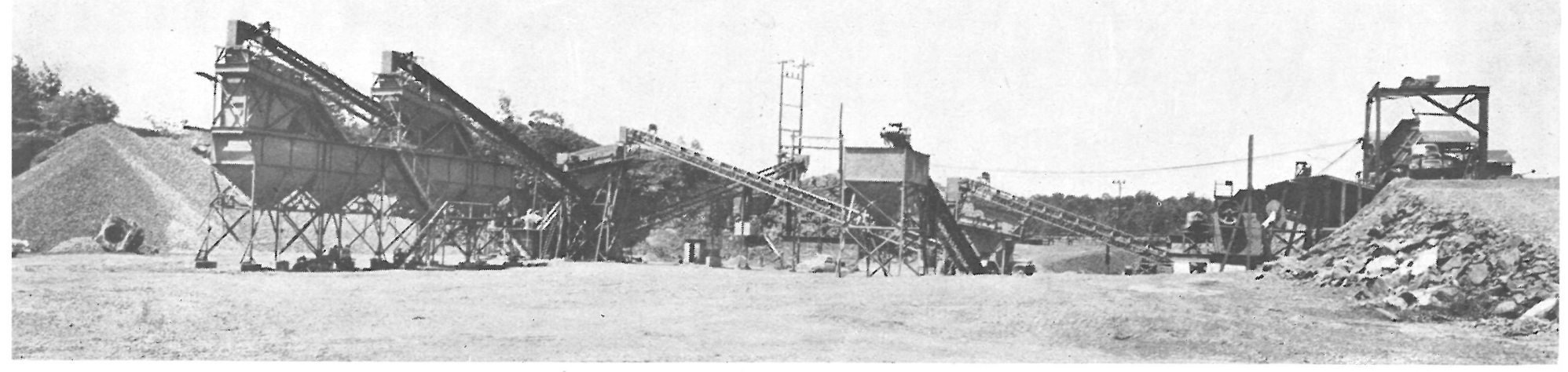

Vista de la instalación mostrando la rampa de alimentación primaria y las pilas de almacenamiento.

Tolva de alimentación de la machacadora primaria, de quijadas, que se halla de la tolva.

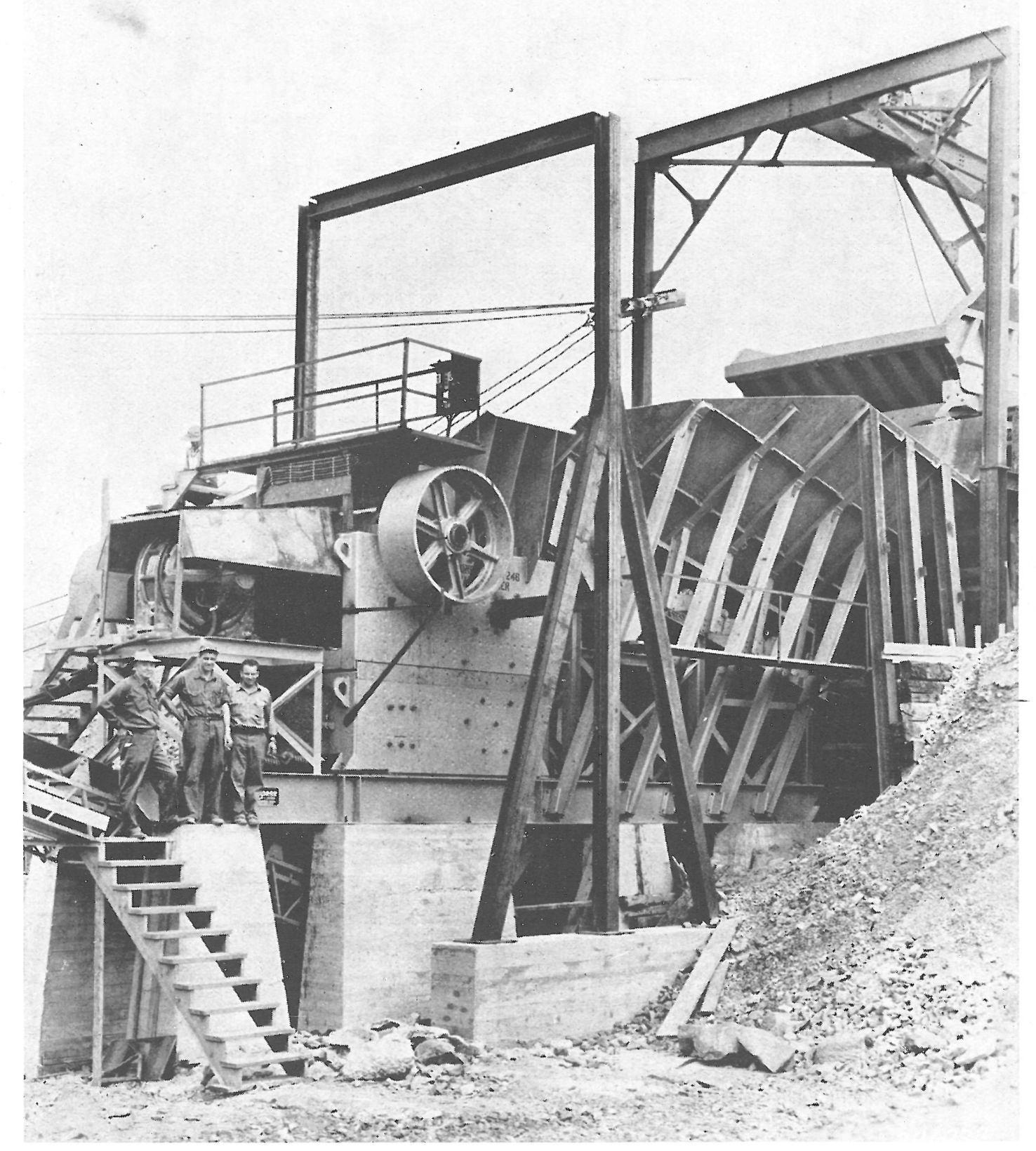




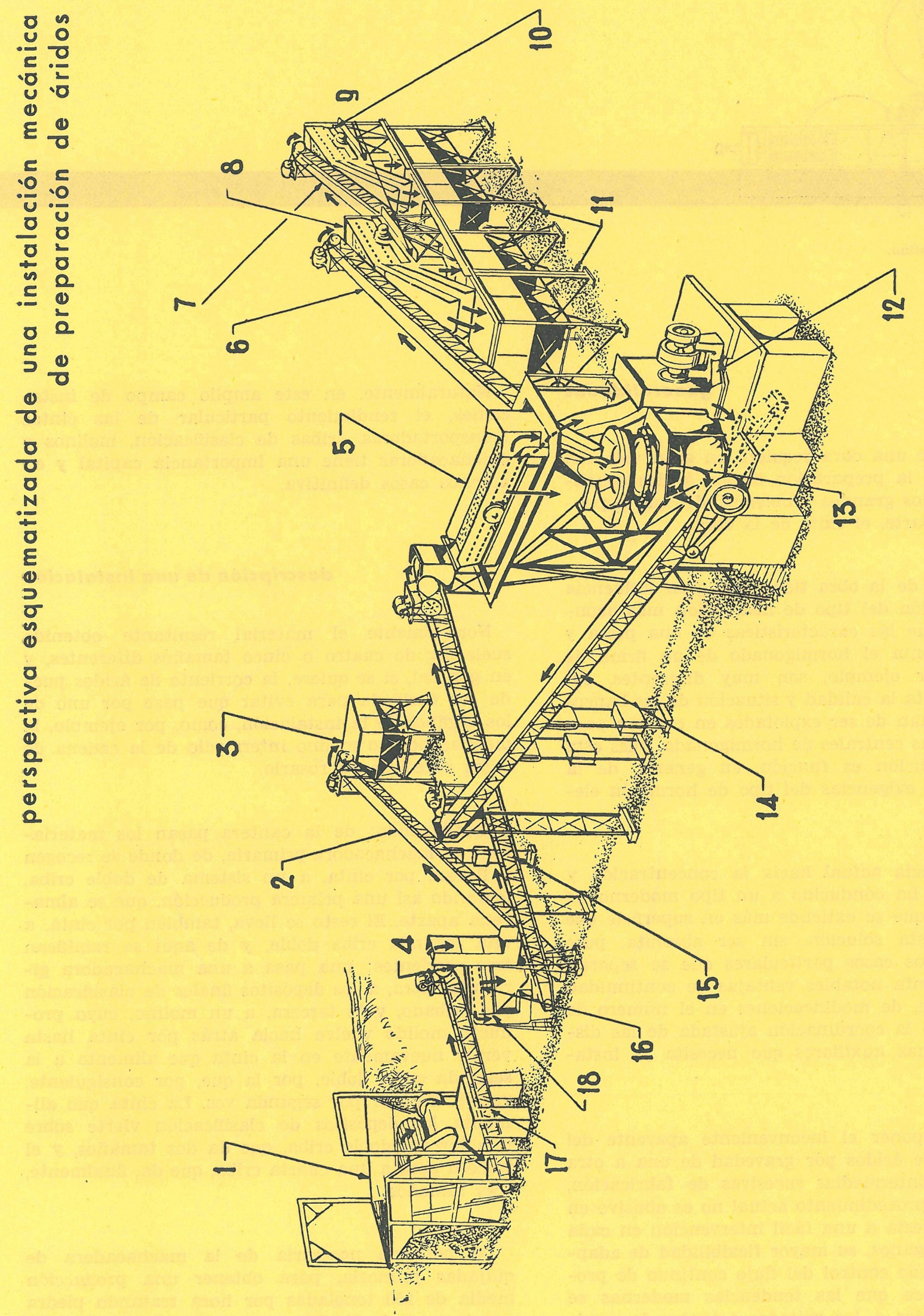

象 
Esquema del molino.

\section{generalidades}

Siempre que una obra requiere un gran volumen de hormigón, la preparación de los áridos constituye uno de los grandes problemas, del que depende, en gran parte, el éxito de la obra.

El carácter de la obra tiene una gran influencia para la elección del tipo de instalación más aconsejable, ya que las características de una presa y las que presenta el hormigonado de un firme de carretera, por ejemplo, son muy diferentes, sin tener en cuenta la calidad y situación de los bancos de roca que han de ser explotados en cantera para alimentar a las centrales de hormigonado, pues esta segunda condición es función, en general, de la localización y exigencias del tipo de hormigón elegido.

Una tendencia actual hacia la concentración y mecanización ha conducido a un tipo moderno de instalaciones que se extiende más en superficie que en altura. Esta solución, sin ser absoluta, pues existen muchos casos particulares que se separan de ella, presenta notables ventajas de continuidad de fabricación, de modificaciones en el número de tamaños y de la coordinación ajustada de las distintas máquinas auxiliares que necesita la instalación.

Se podrá oponer el inconveniente aparente del movimiento de áridos por gravedad de una a otra de las fases intermedias sucesivas de fabricación, pero como el procedimiento actual no es abusivo en altura y se presta a una fácil intervención en cada uno de sus órganos, su mayor flexibilidad de adaptación y cómodo control del flujo continuo de producción, parece que las tendencias modernas se acomodan mejor a los métodos actuales de suministro y fabricación de áridos.
Naturalmente, en este amplio campo de instaciones, el rendimiento particular de las cintas transportadoras, cribas de clasificación, molinos y machacadoras tiene una importancia capital y en muchos casos definitiva.

\section{descripción de una instalación}

Normalmente el material resultante obtenido suele ser de cuatro o cinco tamaños diferentes, y en general, si se quiere, la corriente de áridos puede ser derivada para evitar que pase por uno de los órganos de la instalación, como, por ejemplo, la machacadora o molino intermedio de la cadena de fases sucesivas en rosario.

Sintetizando: de la cantera pasan los materiales a la machacadora primaria, de donde se recogen y llevan, por cinta, a un sistema de doble criba, logrando así una primera producción, que se almacena aparte. El resto se lleva, también por cinta, a una segunda criba doble, y de aquí se ramifican tres corrientes: una pasa a una machacadora giratoria; otra, a los depósitos finales de clasificación por cribado, y la tercera, a un molino, cuyo producto molido vuelve hacia atrás por cinta hasta verter nuevamente en la cinta que alimenta a la segunda criba doble, por la que, por consiguiente, vuelve a pasar por segunda vez. La cinta que alimenta los depósitos de clasificación vierte sobre una tercera doble criba, que da dos tamaños, y el tercero pasa a una cuarta criba, que da, finalmente, tres tamaños.

La potencia necesaria de la machacadora de quijadas primaria, para obtener una producción media de 250 toneladas por hora tratando piedra arenisca, es de $200 \mathrm{HP}$ y un motor de $10 \mathrm{HP}$ para el sistema de alimentación. 


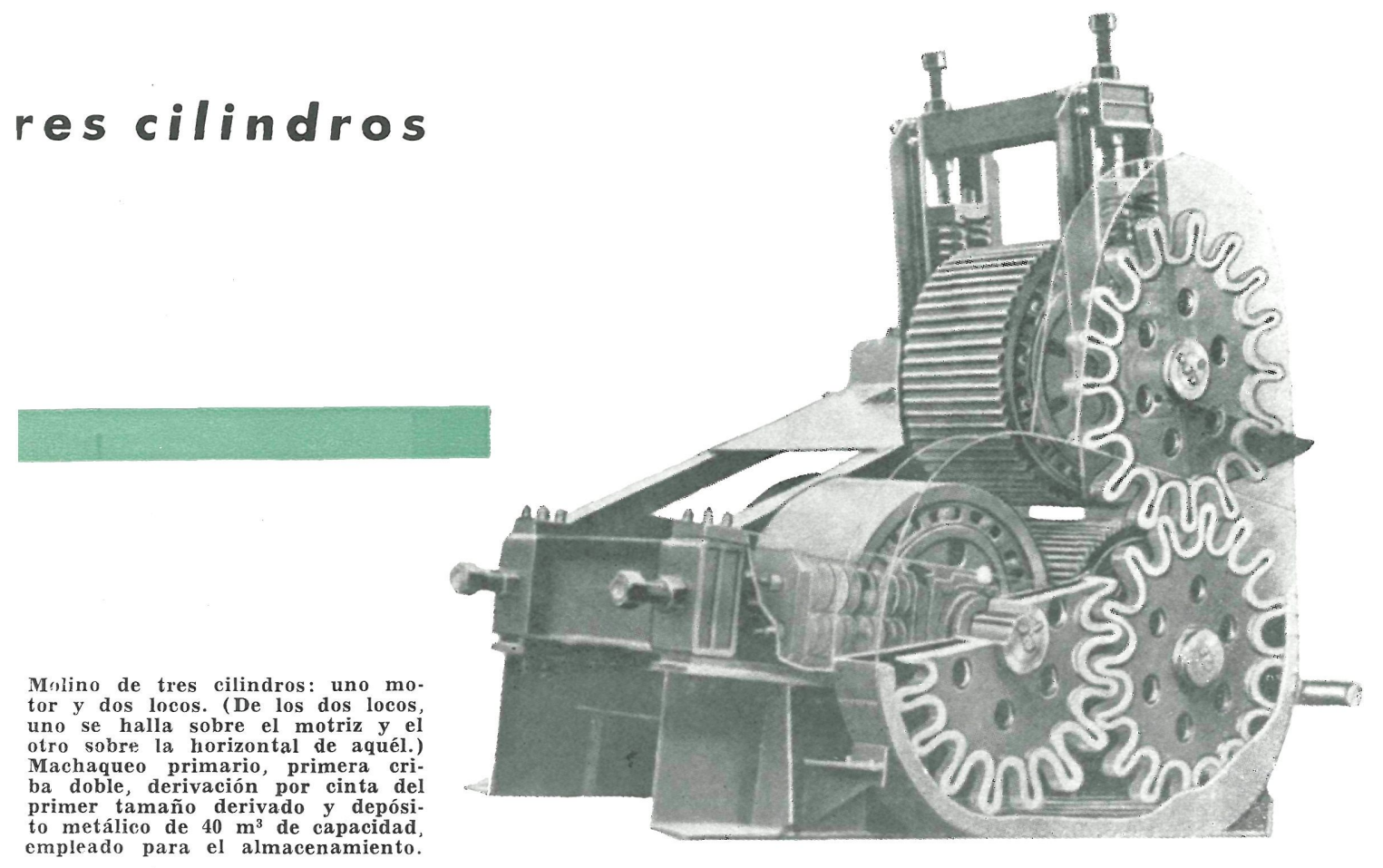

La potencia necesaria para la trituradora giratoria es de $150 \mathrm{HP}$, y de 125 HP para el molino.

Los tamaños obtenidos son de 76 a $50 \mathrm{~mm} ; 50$ a $36 ; 36$ a $25 ; 25$ a 12 , y arena.

Las cribas dobles van equipadas con sus correspondientes compuertas para poder derivar la corriente de áridos que en ellas entre.

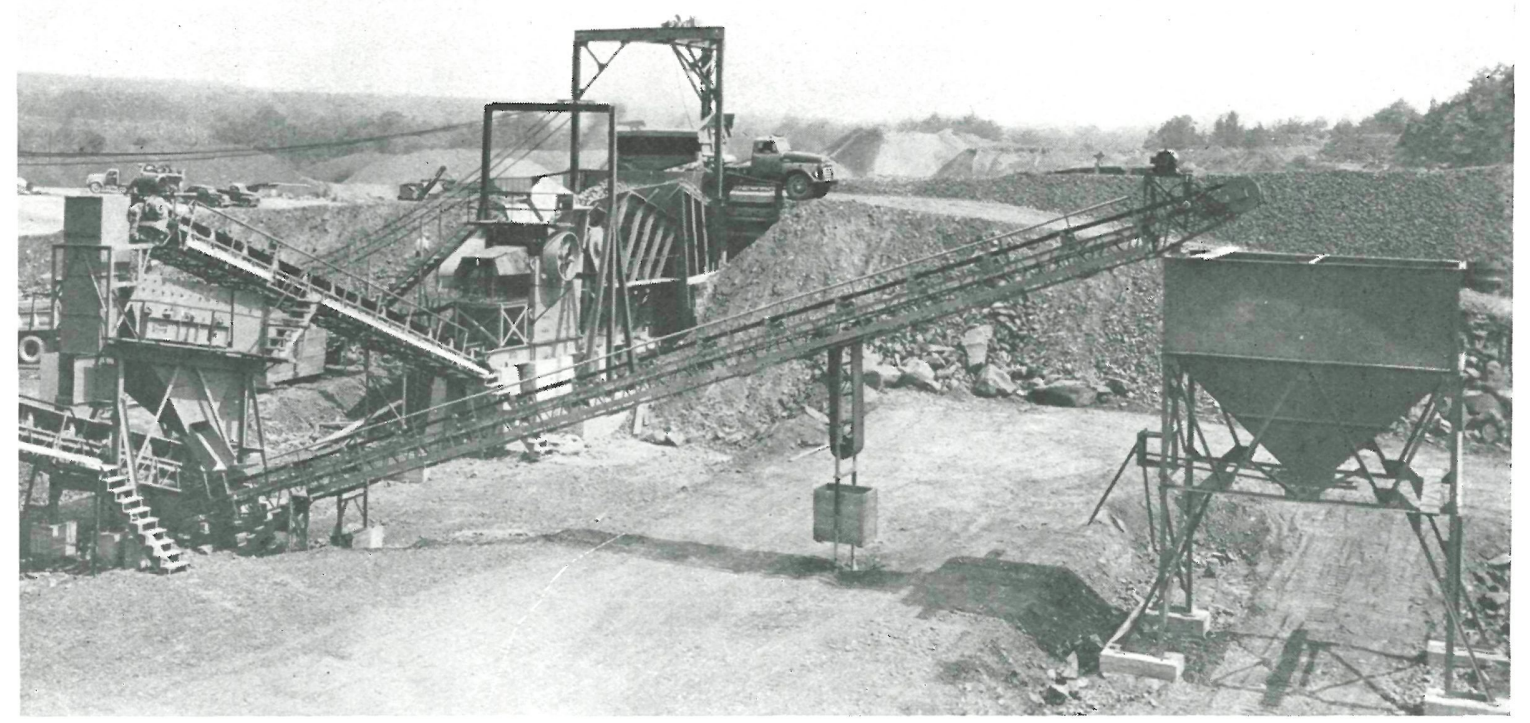

